\title{
On the relationship between the algebraic connectivity and graph's robustness to node and link failures
}

\author{
A. Jamakovic, S. Uhlig \\ Delft University of Technology \\ Electrical Engineering, Mathematics and Computer Science \\ P.O. Box 5031, 2600 GA Delft, The Netherlands \\ \{A.Jamakovic, S.P.W.G.Uhlig\}@ewi.tudelft.nl
}

\begin{abstract}
We study the algebraic connectivity in relation to the graph's robustness to node and link failures. Graph's robustness is quantified with the node and the link connectivity, two topological metrics that give the number of nodes and links that have to be removed in order to disconnect a graph. The algebraic connectivity, i.e. the second smallest eigenvalue of the Laplacian matrix, is a spectral property of a graph, which is an important parameter in the analysis of various robustness-related problems. In this paper we study the relationship between the proposed metrics in three well-known complex network models: the random graph of Erdős-Rényi, the smallworld graph of Watts-Strogatz and the scale-free graph of Barabási-Albert. From [11] it is known that the algebraic connectivity is a lower bound on both the node and the link connectivity. Through extensive simulations with the three complex network models, we show that the algebraic connectivity is not trivially connected to graph's robustness to node and link failures. Furthermore, we show that the tightness of this lower bound is very dependent on the considered complex network model.
\end{abstract}

\section{INTRODUCTION}

Complex networks describe a wide range of systems in nature and society. Traditionally, the topology of complex networks has been modeled as the Erdős-Rényi random graph. However, the growing observation that real-world networks do not follow the prediction of random graphs (e.g. [10]) has prompted many researchers to propose other models, such as small-world [17] and scale-free graphs [1]. Besides the modeling, the analysis of the complex networks' topology in terms of various topological metrics, has attracted considerable attention (see e.g. [2], [16]). The most important include metrics related to degree, clustering, distance, betweenness, connectivity, robustness and graph spectrum.

In this paper we study the relationship between graphs' robustness to node and link failures and the algebraic connectivity, a graph property related to the spectrum of the corresponding Laplacian matrix. The set of eigenvalues of the Laplacian matrix (degree matrix substracted from adjacency matrix equals Laplacian matrix) of a graph is called the Laplacian spectrum [14]. The second smallest eigenvalue of the Laplacian matrix, as proposed by Fiedler in [11], is called the algebraic connectivity. There are many problems in graph theory in which the algebraic connectivity plays a special role (for surveys see e.g. [6], [7], [15]). The most important is its application to the robustness of a graph: 1) the larger the algebraic connectivity is, the more difficult it is to cut a graph into independent components, 2) its classical upper bound in terms of the node and the link connectivity provides worst case robustness to node and link failures [11]. As mentioned in [5], the second means that for every node or link connectivity, there are infinitely many graphs for which the algebraic connectivity is not a sharp lower bound. The node and the link connectivity are important for the robustness because they quantify the extent to which a graph can accommodate to node and link failures. Hence, it is worth investigating the relationship between those three connectivity metrics.

The paper is organized as follows. Section II gives an overview of the Fiedler's algebraic connectivity essentials and the two metrics commonly used to quantify the robustness of a graph, the node and the link connectivity. Section III provides a comprehensive set of simulation results on the relation between the algebraic connectivity and graphs' node and link connectivity in the three wellknown complex network models: the random graph of Erdős-Rényi in Section III.A., the small-world graph of Watts-Strogats in Section III.B., the scale-free graph of Barabási-Albert in Section III.C. and the comparison 
between the three models in Section III.D. Section IV summarizes our main results.

\section{BACKGROUND}

Let $G$ be a graph and let $\mathcal{N}$ denote the set of nodes and $\mathcal{L}$ the set of links, with $N=|\mathcal{N}|$ nodes and $L=|\mathcal{L}|$ links, respectively. The Laplacian matrix of $G$ with $N$ nodes is an $N \times N$ matrix $Q=\Delta-A$ where $\Delta=$ $\operatorname{diag}\left(D_{i}\right), D_{i}$ is the nodal degree of node $i \in \mathcal{N}$ and $A$ is the adjacency matrix of $G$.

The eigenvalues of $Q$ are called the Laplacian eigenvalues. The Laplacian eigenvalues are all real and nonnegative [15]: they are contained in the interval $\left[0, \min \left\{N, 2 D_{\max }\right\}\right]$, where $D_{\max }$ is the maximum nodal degree of $G$. The set of all $N$ Laplacian eigenvalues $\mu_{N}=0 \leq \mu_{N-1} \leq \ldots \leq \mu_{1}$ is called the Laplacian spectrum of $G$. The second smallest Laplacian eigenvalue $\mu_{N-1}$ of $Q$ is known as the algebraic connectivity [11]. As mentioned earlier the algebraic connectivity is very important for the classical connectivity, a basic measure of the robustness of a graph $G: 1$ ) the algebraic connectivity is only equal to zero if $G$ is disconnected, 2 ) the multiplicity of zero as an eigenvalue of $Q$ is equal to the number of disconnected components of $G$.

Let us also introduce two connectivity metrics of $G$ : 1) the link (edge) connectivity $\kappa_{L}$ is the minimal number of links whose removal would disconnect $G, 2$ ) the node (vertex) connectivity $\kappa_{N}$ is defined analogously (nodes together with adjacent links are removed). For $k \geq 1$, a graph $G$ is (node) $k$-connected if either $G$ is a complete graph $K_{k+1}$ or it has at least $k+2$ nodes and no set of $k-1$ nodes that separates it. Similarly, for $k \geq 1$ a graph $G$ is $k$-link connected if it has at least two nodes and no set of at most $k-1$ links that separates it. The maximum value of $k$ for which a connected graph is $k$-connected equals the node connectivity $\kappa_{N}$. The link-connectivity $\kappa_{L}$ is defined analogously [4]. The minimum nodal degree $D_{\min }$ of an incomplete graph $G$ is an upper bound on both the node and the link connectivity $\kappa_{N} \leq \kappa_{L} \leq D_{\min }$. If $G$ is a complete graph $K_{N}$ then $\kappa_{N}=\kappa_{L}=D_{\min }$. Furthermore, the node connectivity of an incomplete graph $G$ is at least as large as the algebraic connectivity $\mu_{N-1} \leq \kappa_{N}$ [11]. If $G=K_{N}$ then $\mu_{N-1}\left(K_{N}\right)=N>\kappa_{N}\left(K_{N}\right)=N-1$. As shown in Figure 1, the relation between the three connectivity metrics is not trivial: $\mu_{N-1}=0.6277 \leq$ $\kappa_{N}=1 \leq \kappa_{L}=2 \leq D_{\min }=3$. Accordingly, the minimal number of nodes $\kappa_{N}$ and the minimal number of links $\kappa_{L}$ to be removed such that no path between any two pairs of nodes remains, in this graph is respectively
1 and 2. Hence, the graph has 1 node-disjoint and 2 linkdisjoint paths. This also means that the depicted graph is 1-node and 2-link connected.

We have used the polynomial time algorithm, explained in [12], to find the node and the link connectivity by solving the maximum-flow problem. The maximumflow problem can be solved with several algorithms, e.g. Edmonds \& Karp [9], Dinic [8], Goldberg [13], etc. If Goldberg's push-relabel algorithm is utilized, as performed in our simulations, the link connectivity algorithm has $O\left(N^{3} \sqrt{L}\right)$-complexity, while the node connectivity algorithm has $O\left(N^{2} L \sqrt{L}\right)$-complexity. We have used the LAPACK implementation of the QRalgorithm for computing all the eigenvalues of the Laplacian matrix. For linear algebra problems involving computation of few extreme eigenvalues of large symmetric matrices, algorithms (e.g. Lanczos) whose run-time and storage cost is lower compared to the algorithms for calculation of all eigenvalues (QR algorithm has $O\left(N^{3}\right)$ complexity) are known [3].

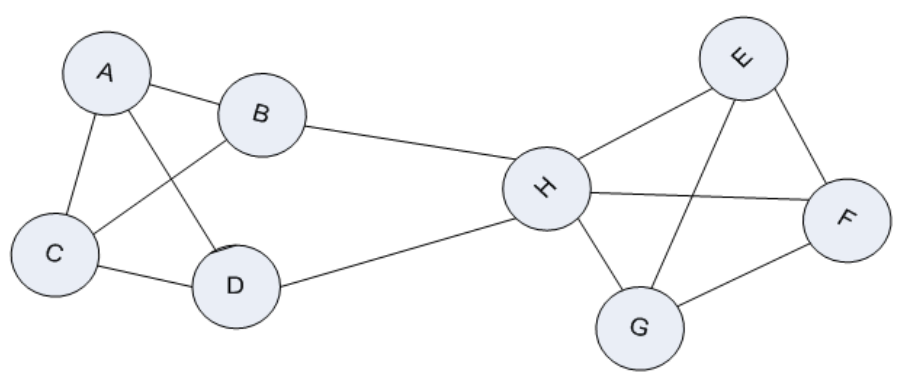

Fig. 1. A graph with $N=8$ nodes and $L=13$ links. The graph's connectivity characteristics are: the node connectivity is 1 (removal of node $\mathrm{H}$ ), the link connectivity is 2 (removal of links connecting node $\mathrm{H}$ to nodes $\mathrm{B}$ and $\mathrm{D}$ ), the algebraic connectivity is 0.6277 and the minimum nodal degree is 3 (minimum number of links a node has).

\section{Simulation Results}

In this section, we present a comprehensive set of simulation results on the relation between three connectivity metrics in generic complex network models: the random graph of Erdös-Rényi, the small-world of WattsStrogatz and scale-free graph of Barabási-Albert. Prior to analyzing the metrics, we define and briefly discuss the models.

\section{A. Random Graph of Erdös-Rényi}

The random graph as proposed by Erdős-Rényi [5] is the simplest model to describe a complex network. In this set of simulations we used the class of random 
graphs denoted by $G_{p}(N)$, in which $N$ is the number of nodes and $p$ the probability of having a link between any two nodes. $G_{p}(N)$ is the set of all such graphs in which the links are chosen independently and the total number of links is on average equal to $p L_{\max }\left(L_{\max }=\frac{N(N-1)}{2}\right.$ is the maximum number of links).

We simulate for each combination of $N$ and $p, 10^{3}$ independent $G_{p}(N)$ graphs. $N$ is 50,500 and 1000 nodes and the link probability $p=\alpha p_{c}$, where ${ }^{1} p_{c}=\frac{\log N}{N}$ and $\alpha$ varies from 1 to 10 . From each combination of $N$ and $p$, we compute the node connectivity $\kappa_{N}$, the link connectivity $\kappa_{L}$ and the algebraic connectivity $\mu_{N-1}$. Then, we classify the simulated graphs according to their value of the node and the link connectivity, which for most graphs have the same value $\kappa_{N}=\kappa_{L}$. Thus, in Figure 2, the mean value (and standard deviation) of the algebraic connectivity is given as a function of both the node and the link connectivity.

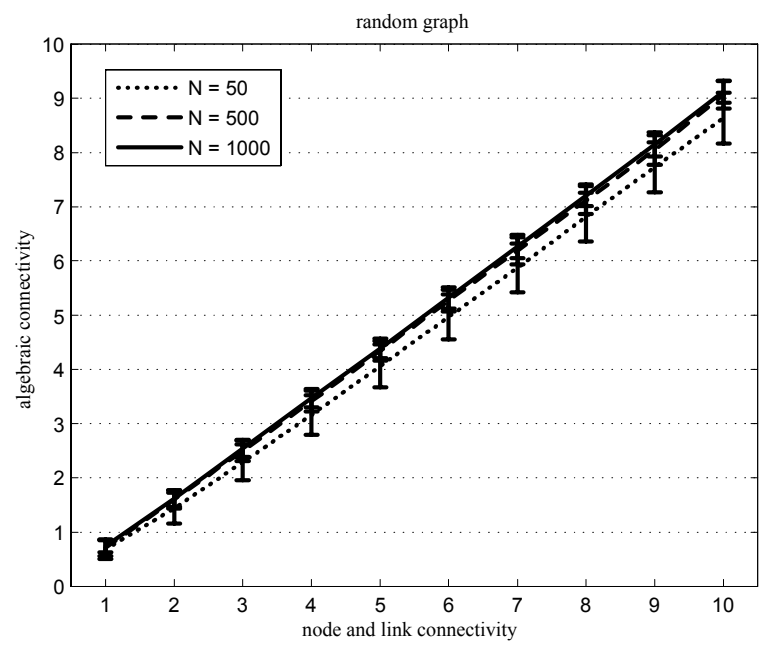

Fig. 2. The mean as well as the standard deviation (error bars) of the algebraic connectivity $\mu_{N-1}$ as a function of the node connectivity $\kappa_{N}$ and the link connectivity $\kappa_{N}$ in the random graph of Erdös-Rényi with $N=50,500$ and 1000 nodes.

The first observation from Figure 2 is that there seems to be a linear relationship between the mean of the algebraic connectivity and the node and the link connectivity. However, from this linear behavior alone, it is not clear whether and how fast the algebraic connectivity converges towards the node and the link connectivity.

In [5], Bollobás proved that irrespective of the link probability $p$, the probability that $\kappa_{N}=\kappa_{L}=D_{\min }$

\footnotetext{
${ }^{1}$ The value of the link probability $p$ above which a random graph almost surely becomes connected tends, for large $N$, to $p \sim p_{c}=$ $\frac{\log N}{N}[5]$.
}

approaches 1 as $N \rightarrow \infty$. Recall that $D_{\min }$ is an upper bound on both $\kappa_{N}$ and $\kappa_{L}$. From Figure 3 we observe that the convergence of $G_{p}(N)$ to a graph where $\kappa_{N}=$ $\kappa_{L}=D_{\min }$ is fast. For example, from the simulation results plotted in Figure 3 with $p=p_{c}$ and a size of the random graph ranging from $N=50$ to $N=1000$, we observe that with probability approaching $1, G_{p}(N)$ has $\kappa_{N}=\kappa_{L}=D_{\min }$ for rather small graph sizes. For all other link probabilities, i.e. $p>p_{c}$, the convergence to $\kappa_{N}=\kappa_{L}=D_{\min }$ occurs for even smaller values of $N$ (see Figure 3 for $p=2 p_{c}$ ). This makes $D_{\min }$ a valuable estimate of the minimum number of nodes or links whose deletion results into a disconnected Erdős-Rényi random graph.

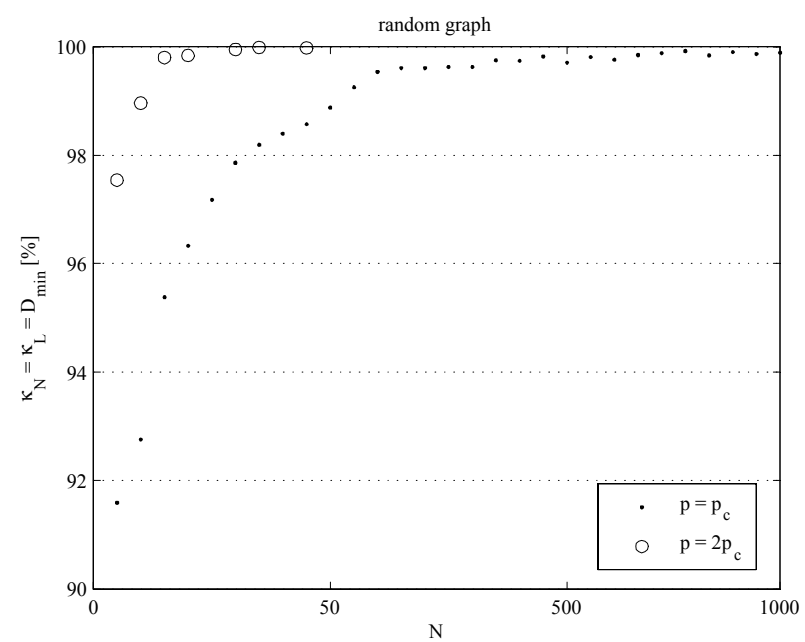

Fig. 3. Percentage of the Erdős-Rényi random graphs with $p=p_{c}$ and $p=p_{c}$ for which the node connectivity $\kappa_{N}$, the link connectivity $\kappa_{L}$ and the minimum nodal degree $D_{\min }$ converge to $\kappa_{N}=\kappa_{N}=$ $D_{\min }$.

Contrary to the convergence of $D_{\min }$, Figure 4 shows that as $N$ tends to large values, the value of $\mu_{N-1}$ does not converge towards $\kappa_{N}$ or $\kappa_{L}$, and obviously not to $D_{\text {min }}$. Furthermore, for a given link density ${ }^{2}$, the difference between $\mu_{N-1}$ and $\kappa_{N}$ or $\kappa_{L}$ is considerable and becomes even more evident if we consider higher values $^{3}$ of the link density $q$ (see Figure 4 for $q=p_{c}$ and $q=2 p_{c}$ ). This behavior is at odds with the one of $D_{\min }$.

\footnotetext{
${ }^{2}$ The link density $q$ in a graph is the fraction between the total and the maximum number of links $q=\frac{L}{L_{\max }}$.

${ }^{3}$ In the Erdős-Rényi random graph, the link density $q$ equals the link probability $p$.
} 


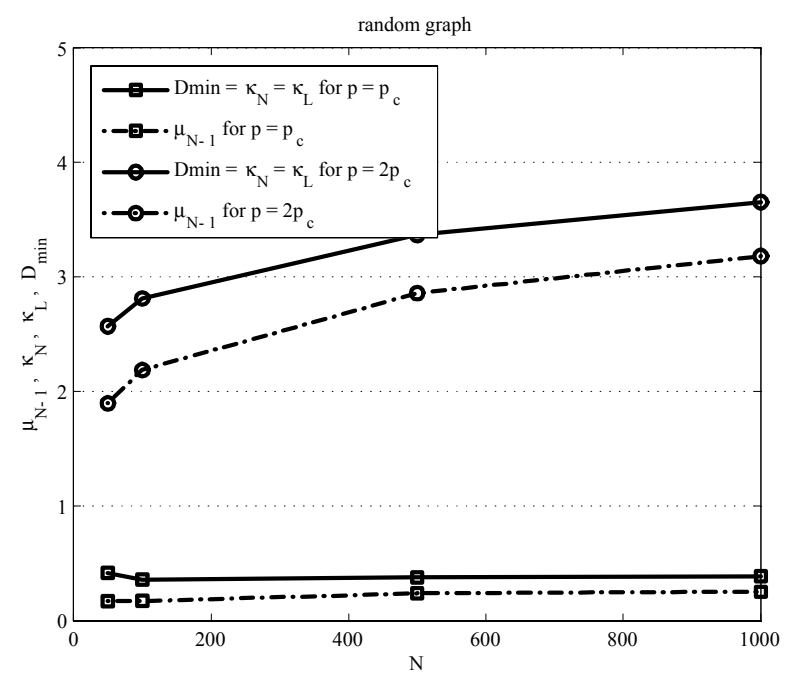

Fig. 4. The mean of the node connectivity $\kappa_{N}$, the link connectivity $\kappa_{L}$ and the algebraic connectivity $\mu_{N-1}$ as a function of the number of nodes $N$ in the Erdös-Rényi random graph with a given link density $q=\frac{L}{L_{\max }}=p$, i.e. $p=p_{c}$ and $p=2 p_{c}$. Also, the minimum nodal degree $D_{\min }$ is depicted as a function of $N$.

\section{B. Small-World Graph of Watts-Strogatz}

The small-world model describes the fact that, despite the large size of the underlying network topology, in most complex networks there is a relatively short path between any two nodes. There are different realizations of the small-world model, but the original model as proposed by Watts and Strogatz [17] is by far the most widely studied. It starts by building the ring $R_{N}$ with $N$ nodes, and then joining each node to $2 s$ neighbors ( $s$ on either side of the ring). This results in the ring lattice $C(N, s)$ with $s N$ links. The small-world graph is then created by moving, with probability $p_{r}$, one end of each link (connected to a clockwise neighbor) to a new node chosen uniformly in the ring lattice, except that no double links or loops are allowed. The rewiring process allows the small-world model to interpolate between a regular lattice $\left(p_{r}=0\right)$ and something which is similar, though not identical, to a random graph $\left(p_{r}=1\right)$. For $p_{r}=1$, it is known that each node has a minimum of $D_{\min }=s$ links [17].

We have simulated, for each combination of $N$ and $s, 10^{3}$ independent Watts-Strogatz small-world graphs. $N$ is 50,100 and 500 nodes and $s$ varies from 1 to 10. The rewiring probability is set to be $p_{r}=1$. From each combination of $N$ and $s$, we compute the node connectivity $\kappa_{N}$, the link connectivity $\kappa_{L}$ and the algebraic connectivity $\mu_{N-1}$. Then, we classify the simulated graphs according to their value of $\kappa_{N}$ and $\kappa_{L}$. Similarly to Figure 2, we plot in Figure 5 the mean (and standard deviation) of the algebraic connectivity as a function of the node and the link connectivity. In most simulated small-world graphs, we observe that $\kappa_{N}=\kappa_{L}$. Hence, the curve depicting the mean (and standard deviation) as a function of the node connectivity turns out to be indistinguishable from the curve for the link connectivity.

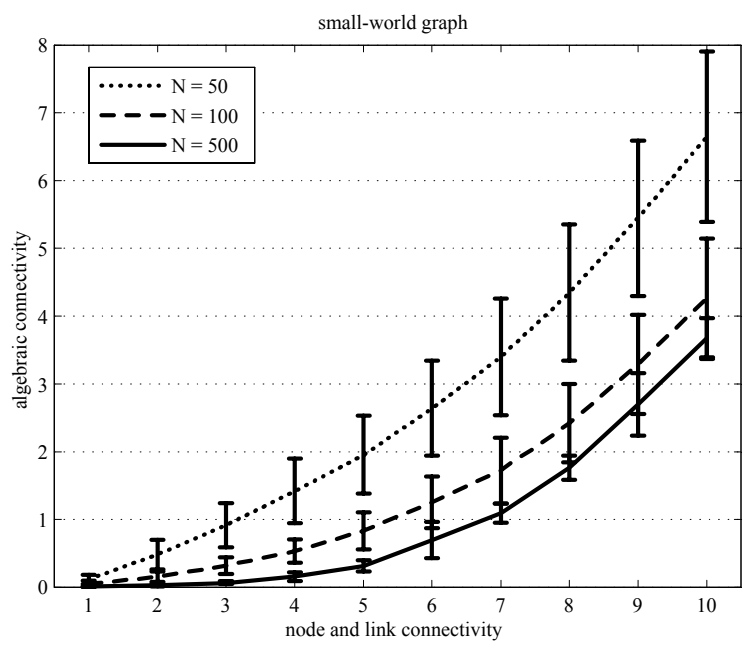

Fig. 5. The mean as well as the standard deviation (error bars) of the algebraic connectivity as a function of the node and the link connectivity in the small-world graph of Watts-Strogatz with $N=50$, 100 and 500 nodes.

From Figure 5 we observe that the algebraic connectivity is a very loose lower bound on the node or the link connectivity. Moreover, the larger the graph size $N$, the looser the bound becomes. This means that for a given value of the node or the link connectivity, the mean value of the algebraic connectivity is a decreasing function of the graph size $N$, opposite to Erdős-Rényi random graph (see Figure 2). Furthermore, the larger the graph size $N$, the smaller the standard deviation (see error bars in Figure 5).

In Figure 5, for a given value of the node or the link connectivity, the algebraic connectivity $\mu_{N-1}$ seemed to be a decreasing function of $N$. However, Figure 6 shows that for small-world graphs with a given link density ${ }^{4}$ $q, \mu_{N-1}$ is an increasing function of $N$. Figure 6 also shows that $\kappa_{N}$ and $\kappa_{L}$ approach $D_{\min }$ for already small $N$. Thus, similarly to Erdős-Rényi random graph, the minimum number of nodes is a valuable estimate of

\footnotetext{
${ }^{4}$ In the Watts-Strogatz small-world graph the link density $q$ is $q=$ $\frac{L}{L_{\max }}=\frac{s N}{L_{\max }}=\frac{2 s}{(N-1)}$.
} 
the minimum number of nodes or links whose deletion results into a disconnected Watts-Strogatz small-world graph. Moreover, the larger the number of neighbors $s$ in the ring lattice (on which the small-world graph is built), the larger the difference seems to be between $\mu_{N-1}$ and $\kappa_{N}$ or $\kappa_{L}$. Recall that in the Watts-Strogatz small-world, each node has a minimum of $D_{\min }=s$ links. Hence, the algebraic connectivity is indicating that as the graph size $N$ increases, the underlying topology of this small-world graph converges to a more robust structure: by expanding $N$ and reducing the link density $q$, it might be possible to increase the number of nodes or link failures and still get the same value of the algebraic connectivity.

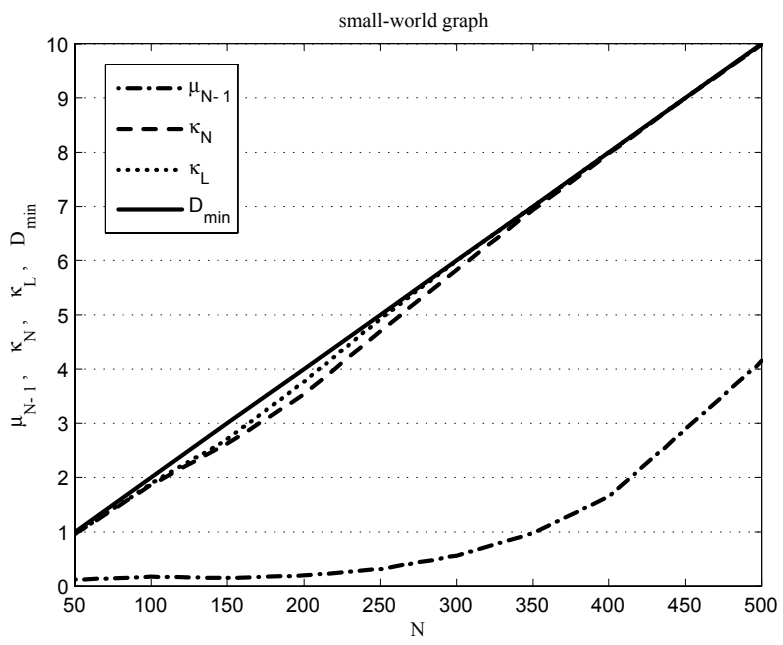

Fig. 6. The mean of the node connectivity $\kappa_{N}$, the link connectivity $\kappa_{L}$ and the algebraic connectivity $\mu_{N-1}$ as function of the number of nodes $N$ in the Watts-Strogatz small-world graph with a given value of the link density $q=\frac{L}{L_{\max }}=\frac{s N}{L_{\max }}=0.04$. The minimum nodal degree $D_{\min }$ equals the number of neighbors $s$ in the ring lattice.

\section{Scale-Free Graph of Barabási-Albert}

Scale-free models have a power-law degree distribution which contrasts with that of random and small-world graphs. Barabási [1] showed that growth and preferential attachment of nodes, which implies that the nodes with larger degree are more likely candidates for attachment of new nodes, give rise to a class of graphs with a powerlaw degree distribution. The Barabási-Albert model starts with a small number $m_{0}$ of fully-meshed nodes, followed at every time step by a new node attached to $m \leq m_{0}=$ $2 m+1$ nodes already present in the system. After $t$ time steps this procedure results in a graph with $N=t+m_{0}$ nodes and $L=\frac{m_{0}\left(m_{0}-1\right)}{2}+m t$ links.

We have simulated, for each combination of $N$ and $m$, $10^{3}$ independent Barabási-Albert scale-free graphs. $N$ is
50, 100 and 500 nodes and $m$ varying from 1 to 10 . In the same way as in the simulations for the Erdoss-Rényi random graph and the Watts-Strogatz small-world, we compute for each combination of $N$ and $m$, the three connectivity metrics. Figure 7 shows the mean of the algebraic connectivity $\mu_{N-1}$, obtained by classifying the generated graphs according to their value of the node connectivity $\kappa_{N}$ and the link connectivity $\kappa_{L}$. The scalefree graph is constructed in such a way that deleting $m$ links or $m$ nodes to which a new node (in the last time step) is attached, leads to $m=\kappa_{N}=\kappa_{L}$. The convergence to a graph where $\kappa_{N}=D_{\min }$ is observed for all combinations of $N$ and $m$. Hence, the mean (and standard deviation) of the algebraic connectivity as a function of the node connectivity is identical to the mean obtained for the link connectivity.

Similarly to the Watts-Strogatz small-world graph, Figure 7 shows that the mean value of the algebraic connectivity is a decreasing function of the graph size $N$. However, the algebraic connectivity of scale-free graphs with a given link density $q$, is an increasing function of $N$. Recall that for all combinations of $N$ and $m$, $m=\kappa_{N}=D_{\min }$. Hence, the link density $q$, for each $N$ and $m$, equals $q=\frac{L}{L_{\max }} \approx \frac{m_{0}^{2}+2 m t}{N^{2}}$. For example, in Figure 7, the following combinations of $N$ and $m$ have approximately the same value of $q$ while the algebraic connectivity is an increasing function of $N$ : for $N=50$ and $m=9, q=0.37$ and for $N=500$ and $m=10, q=$ 0.4 . Thus, the algebraic connectivity indicates that as the link density $q$ increases, the underlying topology of this scale-free graph converges to a more robust structure. It also indicates that by expanding $N$ and reducing the link density $q$, it might be possible to increase the number of nodes or link failures and still get the same value of the algebraic connectivity.

\section{Comparison of Complex Network Models}

Figure 8 shows the mean as well as the standard deviation (error bars) of the algebraic connectivity as a function of the node and the link connectivity in the considered complex network models. Although the scalefree graph of Barabási-Albert has different topological properties, at least in terms of the degree distribution, Figure 8 shows that the relation between the algebraic connectivity and graph's robustness to node and link failures is similar to that in the Erdös-Rényi random graph. This similarity most probably comes from the fact that for both complex network models, the minimum nodal degree is a tight upper bound on the algebraic connectivity, explaining the almost linear relationship 


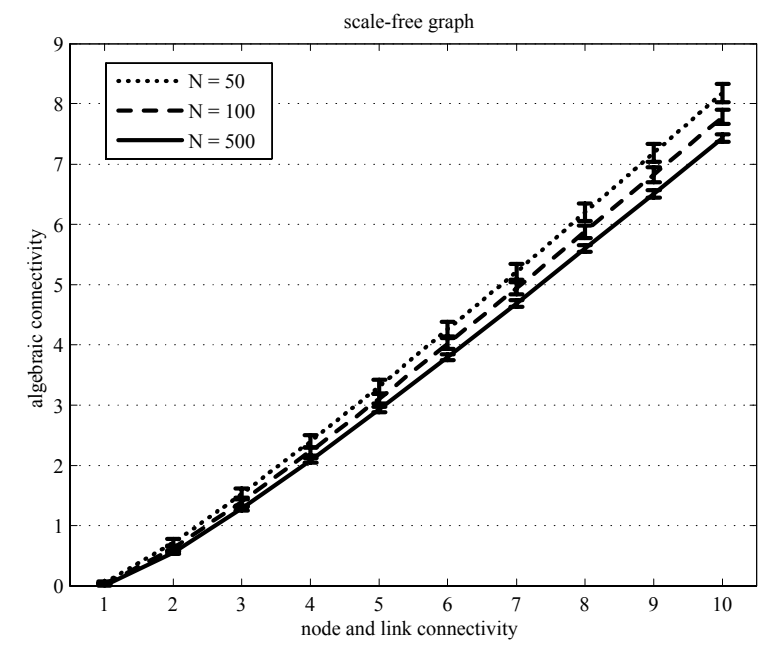

Fig. 7. The mean as well as the standard deviation (error bars) of the algebraic connectivity as a function of the node and the link connectivity in the scale-free graph of Barabási-Albert with $N=50$, 100 and 500 nodes.

between the two connectivity metrics. Recall that we chose the network model parameters so as to perform the simulations within a link density range, which on average results in graphs with a comparable number of links. As shown in Figure 9, the small-world and the scale-free graph, both with a given node or link connectivity, on average have the same number of links. Hence, having the same number of nodes and links, the Barabási-Albert scale-free graph seems to be more robust than the WattsStrogatz small-world graph. For the Erdös-Rényi random graph, the simulations (within a higher link density range than what is used for the other two models) give rise to the larger number of links and therefore possibly the larger value of the algebraic connectivity. Consequently, from the viewpoint of the node and the link connectivity, the robustness of the Erdös-Rényi random graph is worse than the one of the other two complex network models.

\section{Conclusion And Future Work}

In this paper, we have studied Fiedler's algebraic connectivity in relation to the graph's robustness to node and link failures in the following complex network models: random graph of Erdős-Rényi, the small-world of WattsStrogatz and scale-free graph of Barabási-Albert. Based on a comprehensive set of simulations, the following conclusion can be made:

- The algebraic connectivity of a graph increases with the increasing node and the link connectivity (see Figure 8). This means that the larger the algebraic

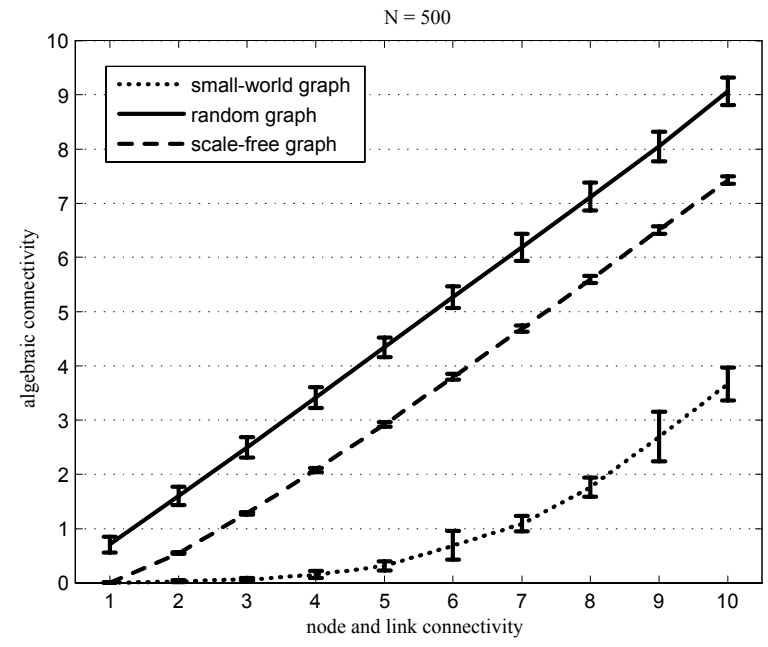

Fig. 8. The mean as well as the standard deviation (error bars) of the algebraic connectivity as a function of the node and the link connectivity in the random graph of Erdös-Rényi, the small-world of Watts-Strogatz and scale-free graph of Barabási-Albert. All graphs have $N=500$ nodes.

connectivity, the larger the number of node- or linkdisjoint paths. Hence, the algebraic connectivity can be considered as a measure of the robustness in complex network models.

- In the three complex network models, the minimum nodal degree is a tight upper bound on both the node and the link connectivity. Hence, the minimum nodal degree is a valuable estimate of the minimum number of nodes or links whose deletion results into a disconnected graph.

- We observe that the relationship between the algebraic connectivity and graph's robustness to node and link failures is not trivial. Even with graph models that have the same number of nodes and links and where the same number of nodes (links) have to be removed to disconnect them, the analyzed connectivity metrics are not trivially related.

Further work comprises studying the algebraic connectivity in other complex network models. This would improve our global understanding of whether and to what extent the algebraic connectivity can be used as a measure of the robustness. The most relevant further work concerns the understanding of how the algebraic connectivity is affected by various structural changes of the network graphs. 


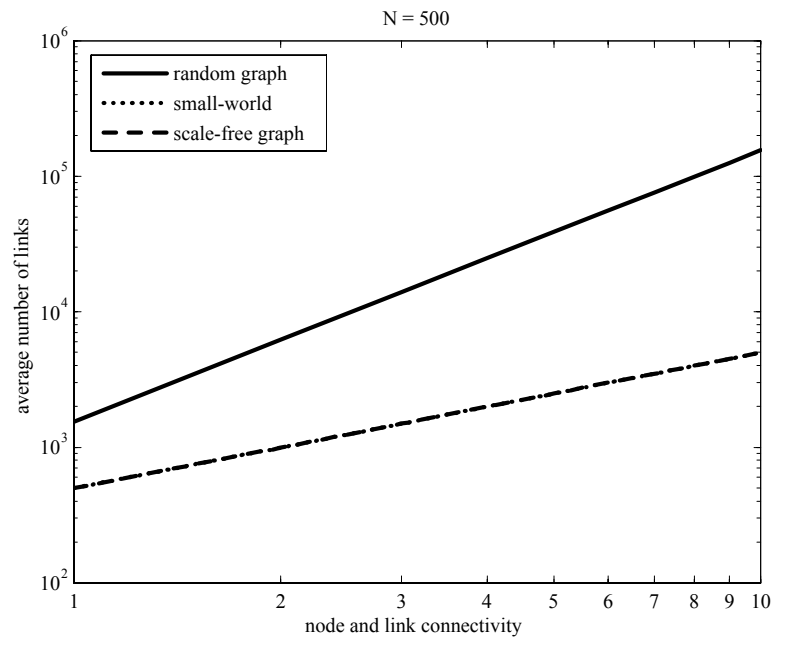

Fig. 9. The average number of links $L$ a considered complex network model has as a function of the node and the link connectivity. For the random graph of Erdős-Rényi, the number of links in a graph with given node or link connectivity is on average equal to $L=p L_{\max }$, for the small-world graph of Watts-Strogatz $L=\frac{2 s}{(N-1)} L_{\max }$ and for the scale-free graph of Barabási-Albert $L=\frac{m_{0}^{2}+2 m t}{N^{2}} L_{\max }$.

\section{ACKNOWLEDGEMENTS}

This research is part of the Next Generation Infrastructures programme www.nginfra.nl, which is partially funded by Dutch government.

\section{REFERENCES}

[1] BARABASI, A.-L. AND ALBERT, R.: Emergence of scaling in random networks, Science 286, pp. 509-512 (1999).

[2] Albert, R., Barabasi, A.-L.: Statistical mechanics of complex networks, Reviews of Modern Physics 74, 47 (2002).

[3] Bai, Z., Demmel, J., Dongarra, J., Ruhe, A. And van DER VORST, H.: Templates for the solution of Algebraic Eigenvalue Problems: A Practical Guide, SIAM, Philadelphia (2000).

[4] BollobÁs, B.: Modern graph theory, Springer-Verlag New York, Inc (2001).

[5] BollobÁs, B.: Random graphs, Cambridge University Press (2001).

[6] Chung, F.R.K.: Spectral graph theory, Conference Board of the Mathematical Sciences 92, American Mathematical Society, Providence, RI (1997).

[7] Cvetković, D.M., Doob, M., Gutman, I. And Torgašev, A.: Recent results in the theory of graph spectra, NorthHolland, Amsterdam (1988).

[8] DiNIC, Y.: Algorithm for solution of a problem of maximumflow in a network, Soviet Mathematics-Doklady 11(5), pp. 1277-1280 (1970).

[9] EDMONDS, J. AND KARP, R.M.: Theoretical improvements in algorithmic efficiency for network flow problems, Journal of the ACM 19(2), pp. 248-264 (1972).

[10] Faloutsos, M., Faloutsos, P. and Faloutsos, C.: On the power-law relationship of the Internet topology, Proceedings of ACM SIGCOMM, pp. 251-262 (1999).
[11] FIEDLER, M.: Algebraic connectivity of graphs, Czechoslovak Mathematical Journal 23, pp. 298-305 (1973).

[12] GibBons, A.: Algorithmic graph theory, Cambridge University Press (1985).

[13] Goldberg, A.V.: A new max-flow algorithm, Technical Memo MIT/LCS/TM-291, MIT Laboratory for Computer Science (1985).

[14] Grone, R., Merris, R., Sunder, V.S.: The Laplacian spectrum of a graph, SIAM Journal on Matrix Analysis 11, pp. 218-238 (1990).

[15] Mohar, B., Alavi, Y., Chartrand, G., Oellermann, O.R. AND SCHWENK, A.J.: The Laplacian spectrum of graphs, Graph Theory, Combinatorics and Applications 2, pp. 871-898 (1991).

[16] Newman, M.E.J.: The structure and function of complex networks, SIAM Review 45, pp.167-256 (2002).

[17] Watts, D.J. And Strogatz, S.H.: Collective dynamics of small-world networks, Nature 393, pp. 440-442 (1999). 